\title{
L'eau vivante et l'eau morte dans l'univers féminin du cinéma tunisien: la mer dans La Saison des hommes (2000) de Moufida Tlatli et la salle de bains dans Les Secrets (2009) de Raja Amari
}

\author{
Cortijo-Talavera, Adela
}

Universitat de València Adela.Cortijo@uv.es

\begin{abstract}
Resumen
Moufida Tlatli y Raja Amari, son dos directoras de cine tunecinas que recurren al elemento acuático como metáfora visual de la construcción de sus universos femeninos particulares. El agua, en tanto que símbolo de impulsión vital y medio de transformación identitaria, a menudo está presente en el imaginario de los cineastas magrebíes. Mis reflexiones se centrarán en dos films concretos en los que existe un tratamiento antitético del agua : Tlatli presenta, en La Saison des hommes (2000), una cartografía del deseo en la que la mirada y la figura femeninas están ligadas a la libertad de un mar mítico y la isla de Djerba. Ella muestra un "ginecine ", en un chronotopos contrastado de cierre y de apertura, donde los hombres, que trabajan en tierra firme, visitan a sus mujeres una vez al año. Y Raja Amar, al contrario, encierra a su grupo de mujeres en una casa acuario de paredes azules, en la que el agua mórbida del cuarto de baño refuerza el sentimiento de angustia.
\end{abstract}

Palabras clave : cine ; Tlatli ; agua ; identidad ; Amari.

\begin{abstract}
Résumé
Moufida Tlatli et Raja Amari, deux réalisatrices tunisiennes qui ont recours à l'élément aquatique en tant que métaphore visuelle de la construction de leurs univers féminins particuliers. L'eau, en tant que symbole d'impulsion vitale et moyen de transformation identitaire, est souvent présente dans l'imaginaire des cinéastes maghrébines. Mes réflexions verseront sur deux films concrets où il existe un traitement antithétique de l'eau : Tlatli présente, dans La Saison des hommes (2000), une cartographie du désir où le regard et la figure féminines sont liés à la liberté d'une mer mythique et à l'île de Djerba. Elle montre un " gynéciné », dans un chronotope contrasté de fermeture et d'ouverture, où les hommes, qui travaillent sur le continent, rendent visite à leurs femmes une fois par an. Et Raja Amar, par contre, enferme son groupe de femmes dans une maison aquarium aux murs bleus, où l'eau morbide de la salle de bains renforce le sentiment d'angoisse.
\end{abstract}

Mots-clés : cinéma ; Tlatli ; eau ; identité ; Amari.

\begin{abstract}
Moufida Tlatli and Raja Amari, are two Tunisian film directors who use the water feature as a visual metaphor for the construction of their particular female universes. Water, while vital drive symbol and means of identity transformation, is often present in the imagination of the Maghreb filmmakers. My reflections will focus on two specific films in which an antithetical water treatment exists : Tlatli presents, in La Saison des hommes (2000), a cartography of desire in which the look and female figure are linked to the freedom of a mythical sea and the island of Djerba. She shows a " ginecine » in a contrasting closing and opening chronotopes, where men who work on the mainland, visit their wives once a year. And Raja Amar, on the contrary, locks his group of women in a home aquarium blue walls, in which the water morbid bathroom reinforces the feeling of anguish.
\end{abstract}

Keywords : cinema ; Tlatli ; water ; identity ; Amari. 
L'eau vivante et l'eau morte dans l'univers féminin du cinéma tunisien : la mer dans La Saison des hommes (2000) de Moufida Tlatli et la salle de bains dans Les Secrets (2009) de Raja Amari

\section{Introduction}

Moufida Tlatli et Raja Amari sont deux réalisatrices tunisiennes qui ont recours à l'élément aquatique en tant que métaphore visuelle de la construction de leurs univers féminins particuliers. L'eau, en tant que symbole d'impulsion vitale et moyen de transformation identitaire, est souvent présente dans l'imaginaire des cinéastes maghrébines. Mes réflexions verseront sur deux films concrets où il existe un traitement antithétique de l'eau: Tlatli présente, dans La Saison des hommes (2000), une cartographie du désir où le regard et la figure féminines sont liés à la liberté d'une mer mythique et à l'île de Djerba. Elle montre un gynéciné, dans un chronotope contrasté de fermeture, la maison de l'île et d'ouverture, la mer. Et Raja Amari, par contre, enferme son groupe de femmes dans une maison aquarium aux murs bleus, où l'eau morte et exigüe de la salle de bains renforce un sentiment d'angoisse et de morbidité.

Les deux affiches des films sont en bleu, mais « le bleu est une couleur chaude » et froide.

Sonia Chamkhi, dans un article paru dans la revue Africultures : «Du discours social au discours de l'intime, ou de la démystification de la violence ${ }^{1}$ ", fait un bref parcours et énumère des femmes tunisiennes cinéastes qui réalisent des films de fiction depuis les années soixante-dix, sans oublier le cinéma documentaire, intéressé surtout à des questions d'anthropologie sociale et culturelle ${ }^{2}$ ) et elle établit que ce qui constitue une unité discursive de la majorité de leurs films c'est l'élaboration d'un discours féministe nourri par une revendication de liberté sinon sociale, au moins intime. La cinéaste pionnière, Selma Baccar, a consacré son film Fatma 75 (1976) au mouvement féministe tunisien, révélant des personnages qui montrent l'émancipation nécessaire de la femme tunisienne malgré leur statut privilégié depuis Bourghiba $^{3}$. La Trace (1988) de Néjia Ben Mabrouk (1949-), Miel et Cendres (1996) de Nadia Farès, Keswa, le Fil perdu (1997) et L'autre moitié du ciel (2008) de Kalthoum Bornaz (1945-), Khochkhach (Fleur d'oubli) (2005) de Selma Baccar (1945-), Satin rouge (2002) ou Les Secrets (2009) de Raja Amari (1971-) et les trois films de Tlatli.

Dans l'imaginaire du cinéma tunisien, en général, l'eau est présente dans la référence inéluctable de la Méditerranée et aussi, bien sûr, du topos exotique du hammam. Le réalisateur Férid Boughedir, par exemple, un des plus célèbres cinéastes tunisiens, nous offre la vue plaisante de la mer des années 60 dans Un été à la goulette, et dans Halfaouine, l'enfant des terrasses, (1990). Les séquences du hammam abondent avec la figure du voyeur de cet univers féminin intime. Bouguédir a recourt souvent à la pulsion de scopophilie freudienne ${ }^{4}$, où la domination masculine transforme les femmes en objets symboliques, dont leur être est un être perçu. Elle a l'effet de les placer dans un état permanent d'insécurité corporelle ou de dépendance symbolique. (Bourdieu, 1998 : 86). Elles existent par et pour le regard des autres. Et la fonction de l'eau là-dessus est évidente, elle est liée au corps-objet féminin, puisque le corps se montre au moment du bain.

Les réalisatrices femmes adoptent aussi, évidemment, des topiques dans leurs films et avec une perspective spatiale fonctionnelle et symbolique, on retrouve les dichotomies des horizons de la mer et des portes et des fenêtres bleues avec les filigranes des jalousies. C'est à dire, l'ouvert et le fermé, la vie publique et l'intimité, d'une façon plus puissante encore.

Le cas de Moufida Tlatli, cette réalisatrice tunisienne connue et primée aux festivals, sert à déchiffrer la fonction et le motif de l'eau dans son gynéciné où les femmes sont objets et sujets, où elles se montrent mais se regardent aussi en même temps et, comble des excès, elles se sentent, se touchent et s'écoutent. Chez Moufida Tlatli, nous trouverons l'écho mythique d’une mer verte accueillante, chaude, liée à la féminité et à la liberté.

\footnotetext{
${ }^{1}$ http://www.africultures.com/php/?nav=article\&no=11073.

${ }^{2}$ Sophie Ferchiou, auteur, en 1970, de Zerda et, en 1971, de Chéchia. Fatma Skandrani, réalisatrice de Médina, ma mémoire ou encore de Kalthoum Bornaz, auteur en 1987 de Trois personnages en quête d'un théâtre. Aujourd'hui encore, le documentaire est relevant dans quelques cinéastes femmes à l'instar de Nadia El Fani, auteur de Ouled Lénine, en 2007.

${ }^{3}$ La société tunisienne, depuis Bourguiba, dès l'indépendance, a entrepris des actions exceptionnelles à l'échelle du monde arabe pour l'émancipation des femmes, entre autres, par l'obligation de la scolarisation des filles, l'interdiction de la polygamie, l'autorisation du divorce et la suppression de l'obligation du port du voile. Il s'agit bel et bien d'un statut singulier où la politique sociale a marqué la vie des femmes tunisiennes bien au-delà du cercle d'une élite.

${ }^{4}$ Laura Mulvey prend de Freud ce concept de scopophilie dans ses deux aspects, actif et passif, et elle l'applique au dispositif filmique (Mulvey, 1999: 154).
} 
Moufida Tlatli est née en 1947, à Sidi Bou Saïd. Issue d’une famille «traditionaliste », elle découvre le cinéma grâce à son professeur de philosophie et fait des études de montage à l'IDHEC ${ }^{5}$ (ancêtre de la Fémis) à Paris, dont elle sort diplômée en 1968. Son parcours est donc similaire à celui de Néjia Ben Mabrouk ou Kalthoum Bornaz, avec qui elle travaillera dans les Baliseurs du désert. Après ce fameux 68, elle revient en Tunisie et travaille dans le montage de plusieurs films dont Omar Gatlato (1976), premier film de l'algérien Merzak Allouache, Les Baliseurs du désert (1984) de Nacer Khémir, Le Cantique des pierres (1990) de Michel Khleifi ou Halfaouine, l'enfant des terrasses (1990) de Férid Boughedir. Ensuite, elle réalise trois films : En 1994, Les Silences du palais, en 2000, La Saison des hommes, et en 2004, le téléfilm Nadia et Sarra.

\section{L'eau vivante dans La saison des hommes (2000) de Moufida Tlatli}

La saison des hommes, comme les deux autres films, c'est une histoire de filiations et de confrontations de générations, des mères, des filles et des grand-mères. Où la protagoniste absolue, la figure forte, l'icône presque sacrée, c'est la mère : Aïcha.

Les réminiscences de harem exotique se retrouvent dans cette histoire qui se passe sur l'île de Djerba, où les hommes qui travaillent sur le continent, à Tunis, rendent visite à leurs femmes une fois par an. Lorsque Aïcha se marie, à 18 ans, avec Saïd, elle lui demande de l'emmener avec lui. Il y consent, à la condition qu'elle lui donne un garçon. Mais elle accouche de deux filles, et elle met plus de dix ans à avoir le garçon si désiré qui est autiste. Aïcha décide de revenir avec sa bellesœur et ses deux filles adultes à Djerba dont la guérison de l'enfant ne peut venir que du tissage des tapis. Et là se produit, encore une fois, la confrontation avec le passé et les souvenirs de la souffrance d'un espace féminin cloîtré, autour de la figure autoritaire et dominante de la belle-mère qui se bat pour ne pas perdre sa place. La saison des hommes décrit un monde de femmes seules, libérées des hommes, mais pas de l'oppression exercée par une belle-mère qui règne avec tyrannie sur le foyer pendant que leurs fils sont absents.

À travers une structure de flash-backs, Tlatli arrive à connecter deux époques et deux univers, tout comme dans le film précédent, Les silences du palais. Et elle fait usage du huis-clos, de l'espace fermé, du sérail, avec un montage et des plans qui jouent avec la spirale et la circularité intérieures ${ }^{6}$ - le cercle de l'accouchement - et extérieures.

Cette atmosphère et ce paysage intime et étranger a un rapport direct avec les femmes qui peuplent l'univers cinématographique de Tlatli. Elles sont envisageables dans une quête identitaire d'indépendance mais aussi en relation ou en communion avec les autres femmes, et elles se construisent par continuité et par opposition avec leurs générations précédentes, surtout avec leurs mères. Il s'agit d'une symbolique de l'eau quasi amniotique. Rappelons l'essai de Camille Lacoste-Dujardin, Des mères contre les femmes (1985), où elle affirmait que dans le Maghreb et dans toutes les cultures méditerranéennes, la féminité s'est identifiée, et même de nos jours, avec la maternité. Et cette figure, clé de voute, de la mère, et la mer baroque, se présente associée avec le passé et la tradition qu'elle sauvegarde. Et dans le cinéma de ces réalisatrices ${ }^{7}$, et de Moufida Tlatli, en concret, elle rappelle la vieille opposition entre « création » et « procréation ».

Moufida Tlatli présente dans ses films une sorte de Carte du Tendre d'interdits symboliques. L'espace reflet le temps qui oscille, qui se balance entre le passé et le présent. Et ce chronotope de fluctuation temporaire se complète avec un prolongement, presque panthéiste, du corps de la femme dans l'espace où la mer condense toute la sexualité ratée de ces mères-épouses enfermées. Dans La saison des hommes, Djerba sert à reproduire le mythe de L'Odyssée, de l'attente de Pénélope qui tisse et qui défait la nuit le linceul de son beau-père Laërte, et qui attend son mari ainsi qu'un Télémaque désiré mais autiste. Dans le film, les femmes chantent quand elles se baignent dans la mer une chanson sur les hommes qu'elles attendent et elles font mention des sirènes. Et, dans un clin d'œil, un mari travaille dans L'Ulysse. Cette île éternelle, connue depuis l'Antiquité et censée d'être traversée par Odyssée dans son périple, devient une île touristique depuis les années 60 .

\footnotetext{
${ }^{5}$ Institut des Hautes Études Cinématographiques de Paris. Créé en 1943 par Marcel Lherbier.

${ }^{6}$ Yuri Lotman fait référence à l'entrée d’un espace fermé et la sortie : « dans la mesure où l'espace fermé peut être interprété comme cave, tombeau, maison, femme (et en conséquence, avec les traits d’obscurité, chaleur, humidité) et l'entrée s'interprète à différents niveaux comme mort, conception, ou retour au foyer (Lotman, 1979 : 188).

${ }^{7}$ Elles supèrent la « dichotomie entre le refus de la maternité en tant que perte de la condition de sujet » (Simone de Beauvoir) et la « célébration de la maternité en tant que source d'un plaisir ineffable » (Julia Kristeva), et accentuent la « contradiction et ambivalence » de la maternité et des liens entre mères et filles (Tubert, 1996).
} 
Les hommes, qui travaillent sur le continent, rendent visite à leurs femmes une fois par an, pendant la saison des hommes, et le micro univers de ces femmes tourne autour de ce moment de rencontre avec le masculin, avec le sexe et avec la possibilité de la maternité. L’idée de renfermement est encore plus exagérée que dans les Silences du palais. Sous la lumière méditerranéenne, avec des palmiers, et le cri assourdissant des cigales, à Djerba, on attend le retour des hommes, qui est vécu comme une fête, ou comme de nouvelles noces pour certaines, mais comme un cauchemar pour d'autres. L'enfermement et la tradition pèsent, la liberté et la modernité font rêver, le passé et le présent se heurtent à l'instar de générations. Il y a soudain, à l'équateur du film un moment de détente, une bulle d'oxygène, de plaisir tout à fait féminin, hors la maison-prison de la belle-mère, quand elles vont à la mer pour nager et laver leurs cheveux teints d’henné.

La réalisatrice montre la mer, dans cette séquence de liberté, de jouissance absolue dans la baignade, en plongée totale, les femmes qui enlèvent le henné dans la mer verte, couleur émeraude, qui font la planche avec leurs habits rougeâtres. Elles se baignent habillées. Elles rient et chantent tandis qu'elles peignent leurs cheveux. À la plage arrivent des amies, « au moins la saison des hommes permet de nous retrouver » disent-elles, et d'autres femmes, plus modernes, qui habitent en France, arrivent et osent même porter un maillot de bain.

La cinéaste réussit, avec ces cadrages en plongée totale, à rendre sensible le sentiment de libération, et en contrepartie la claustration subie par ces femmes. La saison des hommes joue avec une chronologie d'événements qui se tissent avec la notion d'hétérotopie, terme de Foucault paru dans Des espaces autres (1967) ; ainsi qu'avec l'entrée et sortie de l'espace fermé : Cage, maison, île et sexe féminin.

\section{L'eau morte dans Les secrets (2009) de Raja Amari}

Raja Amari est née à Tunis en 1971, elle fait aussi ses études de cinéma à Paris, elle est diplômée de la Fémis en 1998 et elle devient connue avec son premier long-métrage, Satin rouge ${ }^{8}$. En 2004, elle réalise le documentaire Les traces de l'oubli. Et son dernier film c'est Printemps tunisien (2014).

Les secrets est un film bleu foncé presque noir où encore les espaces sont liés de près aux personnages féminins, et en concret, à la marginalité de trois femmes : la jeune Aïcha, Radea et leur mère, cachées dans le sous-sol d'une immense maison délabrée, proche de l'état de ruine, mais d'une belle décadence exotique, avec ses coupoles orientales comme s’il s'agissait d'un palais de rêverie des Mille et une nuits. Symbole d'une Tunisie de carte postale mais qui n'a rien à voir avec le pays d'aujourd'hui. Comme dans le cas de Tlatli, Amari joue avec un espace qui conjugue une temporalité entrecoupée, faite d'écarts, de contrastes ou de sauts du présent au passé. Le film atteste d'une bipolarisation d'un pays à mi-chemin entre les séquelles de la pauvreté - les trois femmes, la triade des sorcières, Hécate tripartite - et les espoirs d'occidentalisation - Selma, la jeune fille bourgeoise, qui porte des talons et qui a un portable.

La triade cloîtrée est en bas, dans un espace étouffant et, en haut, se trouve le couple des jeunes bourgeois. Le cordon ombilical est un escalier en colimaçon, la spirale cauchemardesque, dont les marches sont symboliquement cassées. La vie de ces femmes claquemurées dans un palais arabe de vieille gloire - ou dans une île mythique chez Tlatli - a un rapport avec l'eau dormante à travers la métonymie visuelle de la salle de bains.

Celle d'en haut aux sales carrelages blancs et celle d'en bas, avec des murs peints en bleu. Des salles de bains malpropres, souillées, où on voit la pourriture, les tâches d'humidité et où les troubles de chacune ressortent : où les femmes vomissent, leurs tripes ou leurs aveux, où elles se masturbent avec les robinets qui ne dégoulinent pas, vérifient la virginité de la jeune fille, fument en cachette, se rasent les jambes, avec des plans détail - Aïcha se rase les jambes au début et à la fin du film, et c'est avec cette lame du rasoir qu'elle égorgera sa mère - et, bien sûr, elles se scrutent au miroir. Référence narcissique de l'eau incontournable.

Ce film au féminin où Raja Amari raconte un drame familiale avec des meurtres, se construit avec des lumières froides, avec une photographie bleuâtre, et l'habitat de ces femmes enfermées, qui prennent comme otage une autre femme habillée d'un pull bleu, est une sorte de piscine ou d'aquarium trouble, avec ces murs peints en bleu, irréguliers, fluctuants, ondoyants. Elles nagent et se montrent comme des vieilles baleines, comme des monstres marins au regard malsain du

\footnotetext{
${ }^{8}$ Moufida Tltatli et Raja Amari obtiendront l'une et l'autre des financements pour leurs deux premiers. En 2001, Moufida Tlatli fait partie du jury du Festival de Cannes.
} 
spectateur qui observe avec curiosité ces femmes coupées volontairement du monde et qui établissent des rapports bizarres entre elles.

Les seules ouvertures sont les fenêtres - Selma entre par la fenêtre - ou le petit écran de la vieille télévision, qui, en plan détail, montre la pêche des thons, qui sont sur le point de s'étouffer, de s'asphyxier, hors de l'eau. Une image en ricochet d'elles-mêmes. Les secrets est un film morbide, aquatique, hypnotique où l'eau propre et fraîche n'est pas présente.

Du robinet en panne il ne sort qu'un maigre filet d'eau. Le film commence avec Aïcha qui se lave le matin sans eau, en se léchant comme les chats. Le seul moment de bain est celui de Selma, la jeune femme kidnappée, qui est lavée dans la baignoire par Radea comme si elle était une possession, une poupée, elle est lavée et frottée précisément par la femme qui la tuera plus tard. La salle de bains est le lieu privilégié dans cette piscine, dans cette prison du souterrain, - Aïcha est ligotée par les autres femmes et à côté, sur la table de chevet, il y a verre d'eau enfermée qu'elle fait tomber et qui provoque à leur tour l'enfermement de Selma -. Cette cage bleue, d'eau morte, c'est le foyer de trois femmes qui gardent un secret douloureux de mort, de maternité et de sexualité manquée.

Par conséquence, que ce soit l'eau vivante, primaire, de Tlatli, ou dormante, hypnotique, même absente d'Amari, l'eau exprime chez elles une certaine tension sexuelle. Les deux réalisatrices ont en commun le thème de la condition controversée de la femme, placée entre la tradition et la modernité, ainsi que du désir d’indépendance d'une femme qui a toujours été l’objet passif du désir masculin. L’approche psychanalytique au cinéma, malgré l'effort de Christian Metz, continue à proposer la femme en tant que but et origine du désir phallique, comme la femme rêvée poursuivie et placée toujours au-delà, entrevue et invisible sur un autre décor (Metz, 1977 : 45).

\section{Conclusion}

Selon Marta Segarra, dans Politiques du désir (2007), le désir dans la fiction littéraire et cinématographique est lié à l'éros et le thanatos et il agit d'une manière contradictoire. Le désir questionne, de plus, une certaine conception du sujet qui a été dominant dans la pensée et les discours artistiques. Celle qui considère le sujet comme une entité unitaire, stable et cohérente, avec des tensions mais capable de dominer les pulsions qui essaient de le pousser au-delà de « ce qui est propre de l'homme », puisque ce sujet prototypique est éminemment masculin. Les femmes, selon cette tradition, soumises à la nature, ne sont pas des sujets de désir mais elles sont sujettes à leur désir. Mais, est-ce que cette sorte de désir est-elle représentée autrement chez ces femmes réalisatrices tunisiennes ? Comment s’inscrit dans le cinéma de Moufida Tlatli ou de Raja Amari une image de la femme en tant que sujet de désir qui s’éloigne, ou pas, du stéréotype configuré par les valeurs traditionnelles du patriarcat ? Constamment sous le regard des autres, des hommes et des autres femmes aussi, qui les jugent ou les jalousent, les femmes sont condamnées à expérimenter la distance entre leur corps réel, auquel elles sont enchaînées et le corps idéal, auquel elles essaient de s’approcher (Bourdieu, 1977 : 87). Les personnages féminins de Moufida Tlatli et de Raja Amari ne s'écartent pas de ceci. Elles ressentent le besoin du regard des autres pour se construire et sont conscientes de leur manière de présenter leur corps en spectacle pour le plaisir du regard. Teresa de Laurentis, avec une perspective féministe dans Alice doesn't (1994) fait référence à la représentation de la femme comme spectacle-corps pour être regardé, lieu de sexualité et objet du désir [...] qui trouve dans le cinéma de fiction son expression plus complexe. (de Laurentis, 1994 : 13). Et l'eau rejoigne aussi, entre le mouvement et la pétrification de la femme dans cette dualité sujet-objet, la surface du miroir. Un miroir qui répond à l’idée de Foucault du miroir comme métaphore de la dualité et les contradictions, de l'utopie d’une image qu’on voit mais qui n’existe pas.

\section{Références bibliographiques et filmographiques}

Allouache, Merzak (1976). Omar Gatlato.

AMARI, Raja (2001). Satin rouge.

AMARI, Raja 2009. Les secrets.

BACCAR, Selma (1976). Fatma 75.

BACCAR, Selma (2005). Khochkhach (Fleur d'oubli).

Bataille, George (2011) [1957]. L'érotisme. Paris : Les éditions de minuit. 
L'eau vivante et l'eau morte dans l'univers féminin du cinéma tunisien : la mer dans La Saison des hommes (2000) de Moufida Tlatli et la salle de bains dans Les Secrets (2009) de Raja Amari

Ben MABRouK, Néjia (1988). La Trace.

Bornaz, Kalthoum (1997). Keswa, le Fil perdu.

BoRnaz, Kalthoum (2008). L'autre moitié du ciel.

BougHEDIR, Férid (1990). Halfaouine, l'enfant des terrasses.

BOUGHEDIR, Férid (1996). Un été à la Goulette.

BouRdieu, Pierre (1998). La domination masculine. Paris : éd. du Seuil

FARES, Nadia (1996). Miel et Cendres.

Foucault, Michael (1984) [1967]. « Des espaces autres » in Dits et écrits. Conférence au Cercle d'études architecturales, le 14 mars 1967), dans Architecture, Mouvement, Continuité, n5, octobre 1984, pp. 46-49.

KAPLAN, Ann (1998) [1983]. Las mujeres y el cine. A ambos lados de la cámara. Madrid : Cátedra. col. Feminismos.

KHEMIR, Nacer (1984). Les Baliseurs du désert.

KHLEIFI, Michel (1990). Le Cantique des pierres.

KuHN, Annette (1991) [1982]. Cine de mujeres. Feminismo y cine. Madrid : Cátedra.

Lacoste-Dujardin, Camille (1985). Des mères contre les femmes. Paris : La Découverte. [Las madres contra las mujeres: patriarcado y maternidad en el mundo árabe, Madrid : Cátedra, 1993.]

LAGARDE Y DE LOS Ríos, Marcela (2011). Los cautiverios de las mujeres, Madresposas, monjas, putas, presas y locas. Universidad nacional autónoma de México. Col. Las cosechas de nuestras madres.

LAuRETIS (DE), Teresa (1992). Alicia ya no, feminismo, semiótica, cine. (trad. de Silvia Iglesias). Madrid : Cátedra. Col. Feminismos.

Lotman, Yuri (1979). « The origin of Plot in the Light of Typology » dans Poetics today.

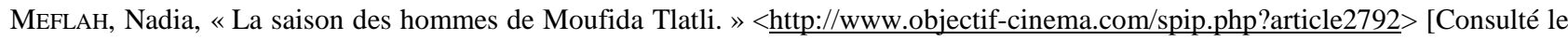
25 juillet 2016].

MERNISSI, Fatima (2001). El harén en occidente. Barcelona : Espasa libros.

METZ, Christian (1977). Le signifiant imaginaire. Paris : UGE.

Mulvey, Laura (1999). « Visual Pleasure and Narrative Cinema » dans Film: Psychologie, Society and Ideology, eds. Leo Braudy and Marshall Cohen. New York : Oxford UP.

Peysson-Zeiss, Agnès (2006). « Chants et vies de femmes dans Les Silences du palais de Moufida Tlatli » dans International Journal of Francophone Studies, volume 9 Issue 2. September.

RolLET, Brigitte. « D’une rive de la Méditerranée à l'autre : financement, diffusion et reconnaissance des réalisatrices du Maghreb »

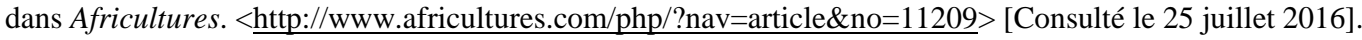

SALHI, Kamal (2007). « Imaging Silence-Representing Women: Moufida Tlatli’s Silences of the Palace and North African Feminist Cinema », dans Quarterly Review of Film and Video. Volume 24, Issue 4, 2007.

SEgARRA, Marta (1998). Mujeres magrebíes. La voz y la mirada en la literatura norteafricana. Barcelona : Icaria.

SegarRa, Marta (2007). Políticas del deseo. Ed. Marta Segarra. Barcelona : Icaria. col. Mujeres y culturas.

SEgARRA, Marta (2008). “¿Las madres contra las mujeres? La maternidad en las novelistas del Magreb », dans Lectora, Revista de dones i textualitat, $\mathrm{n}^{\circ} 14: 65-73$.

SegarRa, Marta (2008). Traces du désir. Paris : Campagne Première.

SEgARRA, Marta (2014). Teoría de los cuerpos agujereados. Madrid : Melusina.

SHERZER, Dina. « Remembrance of Things Past: “Les Silences du palais” by Moufida Tlatli » South Central Review Vol. 17, No. 3, Cinema Engage: Activist Filmmaking in French and Francophone Contexts. (Autumn, 2000), pp. 50-59.

Tlatli, Moufida (1994). Les Silences du palais.

TlatLI, Moufida (2000). La Saison des hommes.

TLATLI, Moufida (2004). Nadia et Sarra.

Tubert, Silvia (1996). Figuras de la madre. Madrid : Cátedra.

ZECCHI, Barbara (2014). La pantalla sexuada. Madrid : Cátedra. col. Feminismos. 